\title{
$S$-wave meson-nucleon scattering in an SU(3) cloudy bag model
}

\author{
E. A. Veit* and B. K. Jennings \\ TRIUMF, 4004 Wesbrook Mall, Vancouver, British Columbia, Canada V6T $2 A 3$ \\ A. W. Thomas \\ Physics Department, University of Adelaide, Adelaide, South Australia 5001 \\ R. C. Barrett \\ Physics Department, University of Surrey, Guildford GU2 5XH, United Kingdom
}

(Received 8 June 1984)

\begin{abstract}
The cloudy bag model (CBM) is extended to incorporate chiral SU(3) $\times \mathrm{SU}(3)$ symmetry, in order to describe $S$-wave $K N$ and $\bar{K} N$ scattering. In spite of the large mass of the kaon, the model yields reasonable results once the physical masses of the mesons are used. We use that version of the CBM in which the mesons couple to the quarks with an axial-vector coupling throughout the bag volume. This version also has a meson-quark contact interaction with the same spin-flavor structure as the exchange of the octet of vector mesons. The present model strongly supports the contention that the $\Lambda^{*}(1405)$ is a $\bar{K} N$ bound state.
\end{abstract}

\section{INTRODUCTION}

Over the past five years the various extensions of the MIT bag model which incorporate PCAC (partial conservation of axial-vector current) have attracted a great deal of interest. ${ }^{1-7}$ This area remains controversial, and issues such as the nucleon bag size, the penetration of the meson field into the bag interior, and the role of the meson field in generating baryon number ${ }^{8-10}$ are still being hotly debated. Nevertheless, these developments have certainly led to some remarkable improvements in our understanding of low-energy hadronic properties (for example, baryon magnetic moments and charge radii, ${ }^{11-15}$ and the axial-vector form factor of the nucleon ${ }^{15-18}$ ) as well as low-energy pion-nucleon scattering ${ }^{7,14,19,20}$ and photoproduction. ${ }^{21}$

Given this interest and success in the pionic sector it seems very natural to consider the extension to chiral $\mathrm{SU}(3) \times \mathrm{SU}(3)$. Several groups have already investigated the corrections to hyperon magnetic moments arising from kaon loops. ${ }^{22,23}$ However, they turned out to be relatively small. We have been motivated both by the discrepancy between the $\bar{K} p$ atomic shift and the $\bar{K} p$ scattering length, ${ }^{24}$ and by the controversial nature of the $\Lambda^{*}(1405)$ to investigate the consequences of a chiral $\mathrm{SU}(3) \times \mathrm{SU}(3)$ extension of the cloudy bag model (CBM) to the low-energy $\bar{K} N$ and $\Sigma \pi$ systems.

Our first major finding, namely, that (as suggested by Dalitz and co-workers for many years $\left.{ }^{25}\right)$ the $\Lambda^{*}(1405)$ is not a simple three-quark state, has already been published as a Letter. ${ }^{26}$ In this paper we shall present a detailed explanation of this result, including the parameter dependence, the tests to which the model has been subjected, and the calculational technique. There is, of course, also some discussion of the physical assumptions on which the calculation is based.

Briefly the structure of the paper is as follows. In Sec. II we define the model, and derive the appropriate Hamiltonian for low-energy $\bar{K} N$ and $\Sigma \pi$ interactions. After some discussion of the approximations made, and the effects of renormalization, we report on some test of the same model for low-energy $K N$ and $\pi N$ scattering. The results for the coupled $\bar{K} N-\Sigma \pi$ system in the region of the $\Lambda^{*}(1405)$ are presented in detail in Sec. III. It will be seen that the model provides an excellent description of the new high-quality data of Hemingway et al. ${ }^{27}$ We reserve Sec. IV for the discussion of several theoretical aspects of the calculation, including the behavior of the $K$ matrices subthreshold, and the fraction of the strength $(\sim 14 \%)$ at the $\Lambda^{*}(1405)$ pole associated with a three-quark state. Finally, in Sec. $V$ we summarize our finding and suggest ways of eliminating some of the approximations used here. We also point the way to some interesting new applications of the model.

\section{FORMAL DEVELOPMENT OF THE MODEL}

The natural generalization of the $\mathrm{SU}(2) \times \mathrm{SU}(2) \mathrm{CBM}$ with volume coupling ${ }^{4,19,20}$ is

$$
\begin{aligned}
L= & (i \bar{q} \not \nabla q-B) \theta_{v}-\frac{1}{2} \bar{q} q \delta_{s}+\frac{1}{2}\left(D_{\mu} \phi\right)^{2} \\
& +\frac{1}{2 f} \bar{q} \gamma^{\mu} \gamma_{5} \lambda \cdot q\left(D_{\mu} \phi\right) \Theta_{v} .
\end{aligned}
$$

Here $q(x)$ and $\phi(x)$ are the quark and meson-octet fields, $B$ is the phenomenological energy density, $f$ is the meson-octet decay constant, and $\lambda$ are the SU(3) matrices of Gell-Mann. The function $\Theta_{v}$ is 1 inside the bag volume and 0 outside, while $\delta_{s}$ is a surface $\delta$ function. For a static, spherical bag, as we assume, these functions reduce to $\theta(R-r)$ and $\delta(R-r)$. The $D$ 's denote the appropriate covariant derivatives.

To make the calculations tractable, it is convenient to do a perturbation expansion of the Lagrangian keeping only the terms up to order $\phi^{2}$. The assumption implicit in this approximation is that the meson fields are rather small or, equivalently, that the bag radius is not small $(\geq 0.8 \mathrm{fm}$ ). To this order in $\phi$ the covariant derivatives reduce to 


$$
\begin{aligned}
& D_{\mu} \phi \simeq \partial_{\mu} \phi, \\
& D q \simeq \partial q+\frac{i}{4 f^{2}} \lambda \cdot\left(\phi \times \partial_{\mu} \phi\right) \gamma^{\mu} q,
\end{aligned}
$$

where the $\mathrm{SU}(3)$ cross product is

$$
\left(\phi \times \partial_{\mu} \phi\right)_{a}=\sum_{b c} \phi_{b} \partial_{\mu} \phi_{c} f_{a b c},
$$

with $f_{a b c}$ being the SU(3) structure constants. ${ }^{28}$ Hence up to order $\phi^{2}$ the Lagrangian density is

$$
\begin{aligned}
L= & (i \bar{q} \partial q-B) \theta_{v}-\frac{1}{2} \bar{q} q \delta_{s}+\frac{1}{2}\left(\partial_{\mu} \phi\right)^{2} \\
& +\frac{\theta_{v}}{2 f} \bar{q} \gamma^{\mu} \gamma_{5} \lambda \cdot q \partial_{\mu} \phi-\frac{\theta_{v}}{4 f^{2}} \bar{q} \lambda \cdot \gamma^{\mu} q\left(\phi \times \partial_{\mu} \phi\right) .
\end{aligned}
$$

The Hamiltonian is obtained in the canonical way from

$$
\hat{H}=\int d^{3} x T^{00}(x),
$$

where $T^{00}$ is the energy-momentum tensor. From Eq. (2.5) we therefore find

$$
\widehat{H}=\hat{H}_{0}+\hat{H}_{s}+\hat{H}_{c},
$$

where $\hat{H}_{0}$ describes free bags and mesons, and the interactions are

$$
\hat{H}_{s}=\int d^{3} x\left(-\frac{\theta_{v}}{2 f}\right) \bar{q} \gamma^{\mu} \gamma_{5} \lambda q \cdot \partial_{\mu} \phi
$$

and

$$
\hat{H}_{c}=\int d^{3} x \frac{\theta_{v}}{4 f^{2}} \bar{q} \lambda \gamma^{\mu} q \cdot\left(\phi \times \partial_{\mu} \phi\right) .
$$

The first-order term $\hat{H}_{s}$ couples a "bare" baryon and a meson to a "bare" baryonic state, while the second-order term $\hat{H}_{c}$ is a contact or four-point interaction.

It is useful to eliminate the spatial derivatives in $\hat{H}_{s}$ by rewriting it as a sum of two terms, one of which contains the surface contribution and the other contains just a time derivative. For this purpose, we do the integration in Eq. (2.8) by parts. Using the Dirac equation, the linear boundary condition on the surface

$$
\text { inq } q=\left.q\right|_{r=R},
$$

where $n^{u}$ is the unit normal to the surface of the confining region, and the relation

$$
\partial_{\mu} \theta_{v}(x)=n_{\mu} \delta_{s}(x),
$$

we get for massless quarks

$$
\hat{H}_{s}=\int\left[\frac{i}{2 f} \bar{q} \gamma_{5} \lambda \cdot q \phi \delta_{s}-\frac{\theta_{v}}{2 f} \partial_{0}\left(\bar{q} \gamma^{0} \gamma_{5} \lambda \cdot q \phi\right)\right] d^{3} x
$$

The case where the strange quark is massive is considered in Appendix A.

The interaction Hamiltonian can be projected onto the space of colorless nonexotic baryon states. ${ }^{4}$ Following Théberge and Thomas, ${ }^{11}$ we write

$$
H=H_{0}+H_{\text {int }}=\sum_{B_{0}, B_{0}^{\prime}} B_{0}^{\dagger}\left\langle B_{0}|\hat{H}| B_{.0}^{\prime}\right\rangle B_{0}^{\prime},
$$

where $B_{0}^{\dagger}\left(B_{0}^{\prime}\right)$ is the creation (annihilation) operator for three quark bags of type $B_{0}\left(B_{0}^{\prime}\right)$ and $\left|B_{0}\right\rangle$ and $\left|B_{0}^{\prime}\right\rangle$ are baryonic bare wave functions. Using the MIT bag wave functions and the Fourier transform of the meson fields,

$\boldsymbol{\phi}(\mathbf{x})=\int \frac{d^{3} k}{\left[(2 \pi)^{3} 2 \omega_{k}\right]^{1 / 2}}\left[a(\mathbf{k}) e^{i \mathbf{k} \cdot \mathbf{x}}+a^{\dagger}(\mathbf{k}) e^{-i \mathbf{k} \cdot \mathbf{x}}\right]$,

where $a$ and $a^{\dagger}$ represent annihilation and creation operators which obey the usual commutation relations, the unperturbed Hamiltonian at the baryon level is

$$
\begin{aligned}
H_{0}= & \sum_{B_{0}}\left(m_{B_{0}}{ }^{2}+k^{2}\right)^{1 / 2} B_{0}^{\dagger} B_{0} \\
& +\sum_{i} \int d^{3} k^{\prime} \omega_{k^{\prime}} a_{i}^{\dagger}\left(\mathbf{k}^{\prime}\right) a_{i}\left(\mathbf{k}^{\prime}\right),
\end{aligned}
$$

where $m_{B_{0}}$ is the MIT bare bag mass. Naturally, the specification of the interaction Hamiltonian depends on the baryons involved in the transition. For the contact piece it is clear that to analyze $K N, \bar{K} N$, and $\pi N$ scattering we need to consider baryon-octet members transitions $\left(B M \rightarrow B^{\prime} M^{\prime}\right)$. For the first-order piece $H_{s}$, since we are primarily interested in low-energy $\bar{K} N$ scattering, we shall consider the transition baryon-meson octet to a baryon $\left(\Lambda^{*}\right)$ composed of $u, d$, and $s$ quarks in an $\mathrm{SU}(3)$ singlet with one quark excited to a $1 p$ level. In this case we need only the $1 s_{1 / 2}$ and $1 p_{1 / 2}$ MIT bag wave functions. For a static spherical bag of radius $R$ the $1 s$ wave function can be written as

$$
q_{1 s}(\mathbf{r}, t)=\frac{N_{s}}{\sqrt{4 \pi}}\left[\begin{array}{c}
j_{0}\left(\omega_{s} r\right) \\
i \sigma \cdot \hat{\mathbf{r}} j_{1}\left(\omega_{s} r\right)
\end{array}\right] e^{-i \omega_{s} t} b \theta(R-r)
$$

where $b$ denotes the spin-isospin wave function of the quark (which can be seen in detail in Ref. 29) and $\omega_{s}=2.04 \cdots / R$ is the energy of the quark ground state which satisfies the linear boundary condition Eq. (2.10). The $1 p_{1 / 2}$ wave function is

$$
q_{1 p_{1 / 2}}(\mathbf{r}, t)=\frac{N_{p}}{\sqrt{4 \pi}}\left[\begin{array}{c}
-\sigma \cdot \hat{\mathbf{r}} j_{1}\left(\omega_{p} r\right) \\
i j_{0}\left(\omega_{p} r\right)
\end{array}\right] e^{-i \omega_{p} t} b \theta(R-r)
$$

with $\omega_{p}=3.81 \cdots / R$ being the energy of the first excited quark state. The normalization factors in Eqs. (2.16) and (2.17) are

$$
N_{s, p}^{2}=\frac{1}{2 j_{0}^{2}\left(\omega_{s, p} R\right) R^{3}} \frac{\omega_{s, p} R}{\omega_{s, p} \mp 1} .
$$

The corresponding wave functions for massive quarks are given in Appendix A.

Using the quantized meson fields and the quark wave functions, the interaction Hamiltonian for the transition $B M \rightarrow \Lambda^{*}$ reduces to 


$$
H_{s}=\sum_{j} \int d^{3} k\left[V_{0 j}(\mathbf{k}) a_{j}(\mathbf{k})+V_{0 j}^{\dagger}(\mathbf{k}) a_{j}^{\dagger}(\mathbf{k})\right],
$$

where $j$ labels the type of meson (including its charge state).

The vertex function is given by

$$
V_{0 j}(\mathbf{k})=B_{0}^{\dagger} v_{0 j}^{B \Lambda^{*}}(\mathbf{k}) \Lambda_{0}^{*}
$$

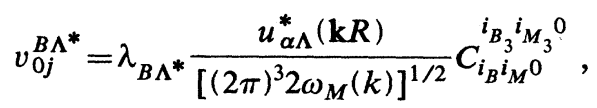

where $\alpha$ labels the meson-baryon pair (e.g., $\bar{K} N$ or $\Sigma \pi$ ), and the dependence of $v_{0 j}$ on $j$ is hidden in the ClebschGordan coefficient. The form factor for $s$-wave scattering is [using Eq. (2.21)]

$u_{\alpha \Lambda^{*}}(k R)=N_{s} N_{p}\left[2 R^{2} j_{0}\left(\omega_{s} R\right) j_{0}\left(\omega_{p} R\right) j_{0}(k R)-\left[\omega_{s}-\omega_{p}+\omega_{M}(k)\right] \int_{0}^{R} d r r^{2}\left[j_{0}\left(\omega_{s} r\right) j_{0}\left(\omega_{p} r\right)+j_{1}\left(\omega_{s} r\right) j_{1}\left(\omega_{p} r\right)\right] j_{0}(k r)\right]$

and the coupling constants are given in Table $\mathbf{I}$.

For $S$-wave scattering the spatial part of the covariant derivative of $\hat{H}_{c}$ [Eq. (2.9)] does not contribute, and we just present the result corresponding to the time derivative for transitions between baryon-meson-octet members:

$$
H_{c}=\sum_{i, j} \int d^{3} k \int d^{3} k^{\prime} V_{0 i j}\left(\mathbf{k}, \mathbf{k}^{\prime}\right) a_{i}^{\dagger}(\mathbf{k}) a_{j}\left(\mathbf{k}^{\prime}\right)
$$

with the vertex function

$$
V_{0 i j}\left(\mathbf{k}, \mathbf{k}^{\prime}\right)=\sum_{B_{0}, B_{0}^{\prime}} B_{0}^{\dagger} v_{0 i j}^{B B^{\prime}}\left(\mathbf{k}, \mathbf{k}^{\prime}\right) B_{0}^{\prime},
$$

where, once again, $(i, j)$ and $\left(B, B^{\prime}\right)$ label the type of meson (and baryon), including its charge state. If we restrict ourselves to purely $S$-wave scattering we find the following explicit expression for $v_{0 i j}^{B B^{\prime}}$ :

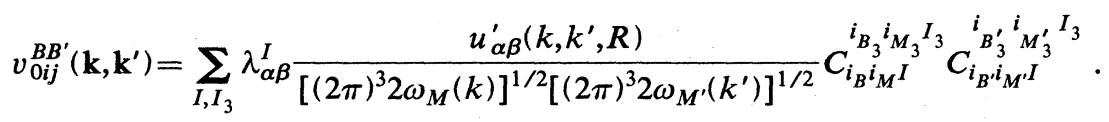

[In Eq. (2.25) $\alpha$ and $\beta$ are a shorthand notation for the initial or final meson-baryon pair-e.g., $\bar{K} N$ or $\Sigma \pi$.] The form factor is

$$
u_{\alpha \beta}^{\prime}\left(k, k^{\prime}, R\right)=N_{s}^{2}\left[\omega_{M}(k)+\omega_{M^{\prime}}\left(k^{\prime}\right)\right] \int_{0}^{R} d r r^{2}\left[j_{0}^{2}\left(\omega_{s} r\right)+j_{1}^{2}\left(\omega_{s} r\right)\right] j_{0}(k r) j_{0}\left(k^{\prime} r\right)
$$

and the coupling constants are given in Tables I and II.

\section{A. Vertex renormalization}

So far the Hamiltonian which we have written down connects bare baryons [see Eq. (2.13)]. However, we want to describe physical baryons which in the CBM consist of "dressed" bags. That is, the physical baryon $B$ is part of the time $\left(Z_{2}^{B}\right)$ a bare three-quark bag, a bare bag surrounded by a cloud of one meson, two mesons, and so on. Thus the physical baryon satisfies the equation ${ }^{11}$

TABLE I. The coupling constants for $\bar{K} N$ scattering with isospin 0 and 1 . In the first column are the values for $2 f \lambda_{\alpha \Lambda^{*}}$ Eq. (2.21), while the other columns contain $2 f^{2} \lambda_{\alpha \beta}^{I}$ Eq. (2.25).

\begin{tabular}{ccccccc}
\hline \hline$I$ & & 0 & & & 1 & \\
& $\Lambda^{*}$ & $\bar{K} N$ & $\pi \Sigma$ & $\bar{K} N$ & $\pi \Sigma$ & $\pi \Lambda$ \\
\hline $\bar{K} N$ & $\sqrt{2}$ & $-\frac{3}{2}$ & $-\sqrt{6} / 4$ & $-\frac{1}{2}$ & $-\frac{1}{2}$ & $\sqrt{6} / 4$ \\
$\pi \Sigma$ & $\sqrt{3}$ & $-\sqrt{6} / 4$ & -2 & $-\frac{1}{2}$ & -1 & 0 \\
$\pi \Lambda$ & 0 & 0 & 0 & $\sqrt{6} / 4$ & 0 & 0 \\
\hline \hline
\end{tabular}

$$
H|B\rangle=m_{B}|B\rangle,
$$

and can be expanded in terms of the bare bag states $\left\{B, B^{\prime}, \ldots\right\}$ :

$$
|B\rangle=\sqrt{Z_{2}^{B}}\left|B_{0}\right\rangle+\Lambda|B\rangle,
$$

where

$$
\Lambda=1-\sum_{B_{0}^{\prime}}\left|B_{0}^{\prime}\right\rangle\left\langle B_{0}^{\prime}\right|
$$

TABLE II. The coupling constants for $S$-wave $K N$ and $\pi N$ scattering with isospin $I$. The values quoted are $2 f^{2} \lambda_{\alpha \beta}^{I}$ in the notation of Eq. (2.25).

\begin{tabular}{ccccc}
\hline \hline$I$ & 1 & 0 & $\frac{3}{2}$ & $\frac{1}{2}$ \\
& $K N$ & $K N$ & $\pi N$ & $\pi N$ \\
\hline$K N$ & 1 & 0 & 0 & 0 \\
$\pi N$ & 0 & 0 & $\frac{1}{2}$ & -1 \\
\hline \hline
\end{tabular}


projects out only those states which contain at least one meson. The mass of the dressed state, $m_{B}$, is then

$$
m_{B}=m_{B_{0}}+\Sigma^{B} \text {. }
$$

In terms of this state $\Sigma^{B}$, the normalization condition on $|B\rangle$ implies

$$
\begin{aligned}
Z_{2}^{B}\left(m_{B}\right) & =\left[1-\frac{\partial}{\partial E} \Sigma^{B}(E)\right]_{E=m_{B}}^{-1} \\
& =\left[1+\Sigma_{B^{\prime}} Z_{2}^{B}\left(B^{\prime}\right)\right]^{-1}
\end{aligned}
$$

The lowest-order self-energy diagram which contributes to $Z_{2}^{B}\left(B^{\prime}\right)$ is shown in Fig. 1 .

Usually the vertex renormalization is discussed in the context of Yukawa couplings, where the renormalized vertex function is

$$
v_{j}^{B B^{\prime}}(\mathbf{k})=\left\langle B\left|V_{0 j}(\mathbf{k})\right| B^{\prime}\right\rangle .
$$

This implies that

$$
v_{j}^{B B^{\prime}}(\mathbf{k})=\frac{\sqrt{Z_{2}^{B}} \sqrt{Z_{2}^{B^{\prime}}}}{Z_{1}^{B B^{\prime}}} v_{0 j}^{B B^{\prime}}(\mathbf{k}),
$$

with the vertex renormalization constant given by

$$
\left(Z_{1}^{B B^{\prime}}\right)^{-1}=1+\sum_{C, D} Z_{1}^{B B^{\prime}}(C, D) .
$$

The lowest-order diagram which contributes to $v_{0 j}^{B B^{\prime}}$, namely, $Z_{1}^{B B^{\prime}}(C, D)$ is shown in Fig. 2(a).

Hence to calculate the vertex renormalization for a $\mathrm{Yu}$ kawa coupling it is necessary to calculate the probabilities $Z_{2}^{B, B^{\prime}, \ldots}$ [defined in Eqs. (2.28) and (2.31)] and the functions $Z_{1}^{B, B^{\prime}}(C, D)$. All of these quantities are given in great detail by Théberge and Thomas. ${ }^{11}$ We shall briefly indicate how to use a similar procedure to calculate the vertex renormalization of the contact interaction. Once again there is a bare interaction:

$$
V_{0 j}^{B B^{\prime}}\left(\mathbf{k}, \mathbf{k}^{\prime}\right)=\left\langle B_{0 i}(\mathbf{k})\left|V_{0}\right| B_{0 j}^{\prime}\left(\mathbf{k}^{\prime}\right)\right\rangle,
$$

which is simply the matrix element of $\mathrm{H}_{2}$ between the initial and final one-meson one-bag states. The renormalized contact interaction is then the matrix element of $\mathrm{H}_{2}$ between physical baryon states. For a given total isospin and angular momentum (labels suppressed for clarity) we can write

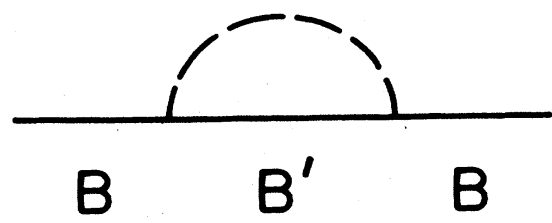

FIG. 1. Lowest-order self-energy diagram for a baryon $B$. A sum must be made over intermediate baryons $B^{\prime}$.

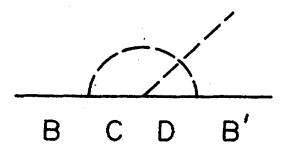

(a)

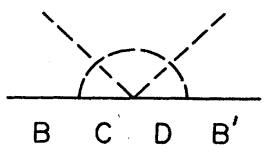

(b)
FIG. 2. Lowest-order diagrams contributing to (a) $v_{0 j}^{B B^{\prime}} Z_{1}^{B B^{\prime}}(C, D)$ [Eq. (2.34)] and to (b) $V_{0 i j}^{B B^{\prime}}\left(\mathbf{k}, \mathbf{k}^{\prime}\right) Z_{1 M M^{\prime}}^{B B^{\prime}}(C, D)$ [Eq. (2.37)].

$$
\begin{aligned}
V_{i j}^{B B^{\prime}}\left(\mathbf{k}, \mathbf{k}^{\prime}\right) & =\left\langle B\left|V_{0 i j}^{B B^{\prime}}\left(\mathbf{k}, \mathbf{k}^{\prime}\right)\right| B^{\prime}\right\rangle \\
& =\frac{\sqrt{Z_{2}^{B}} \sqrt{Z_{2}^{B^{\prime}}}}{Z_{1 \alpha \beta}} V_{0, i j}^{B B^{\prime}}\left(\mathbf{k}, \mathbf{k}^{\prime}\right),
\end{aligned}
$$

where

$$
\left(Z_{1 \alpha \beta}\right)^{-1}=1+\sum_{\gamma, \delta} Z_{1 \alpha \beta}(\gamma, \delta) .
$$

The lowest-order diagram which contributes to $V_{0 i j}^{B B^{\prime}}\left(\mathbf{k}, \mathbf{k}^{\prime}\right)$, namely, $Z_{1 \alpha \beta}(\gamma, \delta)$, is shown in Fig. 2(b). In practice, we have included only the dressing associated with virtual pions. However, we have checked that those graphs involving virtual kaons are small.

Basically the difference between the renormalization of a Yukawa interaction and a contact interaction resides in the difference between Figs. 2(a) and 2(b). As the contact interaction is independent of spin, under certain assumptions it is possible to relate the two renormalizations $Z_{1}$ and $Z_{2}$. For example, if we retain only pion loops, and neglect the $\pi \Lambda \rightarrow \pi \Sigma$ contact interaction inside Fig. 2(b), we find

$$
\left(Z_{1 \alpha \beta}\right)^{-1}=1+\sum_{B^{\prime}} Z_{1 \alpha \beta}\left(B^{\prime}\right)
$$

where for elastic scattering

$$
Z_{1 \alpha \beta}\left(B^{\prime}\right)=Z_{2}^{B}\left(B^{\prime}\right) \frac{\sum_{i_{B}}\left(C_{i_{B^{i}} i_{\pi} i_{B}}^{i_{B^{\prime}}^{i} i_{3} i_{B}}\right)^{2} H_{\mathrm{int}}\left(i_{B^{\prime}}, i_{M}\right)}{H_{\mathrm{int}}\left(i_{B}, i_{M}\right)},
$$

and

$$
H_{\text {int }}\left(i_{B^{\prime}}, i_{M}\right) \propto i_{B_{3}} i_{M_{3}}+\frac{3}{4} Y_{B} Y_{M}
$$

In Eq. (2.40) $i_{B}\left(i_{M}\right)$ is the isospin of the baryon (meson) and $Y_{B}\left(Y_{M}\right)$ the corresponding hypercharge.

Let us consider $K^{+} p$ scattering as an example. Writing the renormalization factors explicitly, we have

$$
\begin{aligned}
V_{K^{+} K^{+}}^{p p}\left(\mathbf{k}, \mathbf{k}^{\prime}\right) & =\left[\frac{1+\frac{2}{3} Z_{2}^{N}(N)+\frac{7}{6} Z_{2}^{N}(\Delta)}{1+Z_{2}^{N}(N)+Z_{2}^{N}(\Delta)}\right] V_{0^{+}{ }^{+}}^{p p}\left(\mathbf{k}, \mathbf{k}^{\prime}\right) \\
& =\mathscr{R}^{K^{+}{ }^{+} V_{0_{K}^{+} K^{+}}^{p p}\left(\mathbf{k}, \mathbf{k}^{\prime}\right)}
\end{aligned}
$$


TABLE III. The renormalized value of $f\left(f_{\text {eff }} / f\right)$ for the different channels. The renormalization is independent of isospin for both the $\pi N$ and $\pi \Sigma$ channels.

\begin{tabular}{lccccc}
\hline \hline & & & $K N$ & \multicolumn{2}{c}{$\bar{K} N$} \\
& $\pi N$ & $\pi \Sigma$ & $I=1$ & $I=0$ & $I=1$ \\
\hline$R=0.8 \mathrm{fm}$ & 1.04 & 1.11 & 1.01 & 1.02 & 0.98 \\
$R=1.0 \mathrm{fm}$ & 1.03 & 1.07 & 1.01 & 1.01 & 0.99 \\
\hline \hline
\end{tabular}

and

$$
\begin{aligned}
& V_{K^{+} K^{+}}^{n n}\left(\mathbf{k}, \mathbf{k}^{\prime}\right)=\left(\frac{1+\frac{5}{3} Z_{2}^{N}(N)+\frac{2}{3} Z_{2}^{N}(\Delta)}{1+Z_{2}^{N}(N)+Z_{2}^{N}(\Delta)}\right) V_{0_{K^{+} K^{+}}^{n n}}\left(\mathbf{k}, \mathbf{k}^{\prime}\right) \\
& =\mathscr{R}^{K^{+}{ }^{n}} V_{0_{K^{+} K^{+}}^{n n}}^{n n}\left(\mathbf{k}, k^{\prime}\right) .
\end{aligned}
$$

Using the results quoted by Théberge and Thomas ${ }^{11}$ for $Z_{2}^{B}\left(B^{\prime}\right)$, the renormalization factors are $\mathscr{R}^{K^{+} p}=0.99$ and $\mathscr{R}^{K^{+}}=1.03$ for the bag radius $R=1 \mathrm{fm}$. It is interesting to note that the renormalization for $K^{+} p$ and $K^{+} n$ is different, this causes the isospin-zero $\mathrm{K}^{+}{ }_{n}$ interaction to become nonzero with strength $a_{0} \simeq a_{1} / 20$ for $R=1 \mathrm{fm}$.

Table III contains the renormalization factors needed in the present work. We quote the effective value for $f$, which is related to $\mathscr{R}$ through

$$
\left[\frac{f_{\text {eff }}}{f}\right)^{2}=\frac{1}{\mathscr{R}}
$$

\section{B. The scattering problem}

In order to solve the scattering problem we define a potential and solve the Lippmann-Schwinger equation. The effect of crossed meson lines is relatively small for pionnucleon scattering. ${ }^{14}$ For kaon-nucleon scattering it is even smaller and for this reason we do not include it here. (In Sec. III we give an estimate of its influence on the $\bar{K} N$ scattering length.) Thus we define the following potential:

$$
\begin{aligned}
v_{\alpha \beta}= & \sum_{B_{0}^{\prime}}\left\langle\alpha\left|H_{s}\right| B_{0}^{\prime}\right\rangle \frac{1}{E-M_{0}\left(B_{0}^{\prime}\right)}\left\langle B_{0}^{\prime}\left|H_{s}\right| \beta\right\rangle \\
& +\left\langle\alpha\left|H_{c}\right| \beta\right\rangle
\end{aligned}
$$

where $|\alpha\rangle$ and $|\beta\rangle$ stand for baryon-meson states and $\left|B_{0}^{\prime}\right\rangle$ is a baryon bag state with mass $M_{0}\left(B_{0}^{\prime}\right)$. This potential is iterated in the Lippmann-Schwinger equation:

$$
\begin{aligned}
t_{\alpha \beta}\left(\mathbf{k}, \mathbf{k}^{\prime}, E\right)= & v_{\alpha \beta}\left(\mathbf{k}, \mathbf{k}^{\prime}, E\right) \\
+ & +\int v_{\alpha \gamma}\left(\mathbf{k}, \mathbf{k}^{\prime \prime} ; E\right) \frac{1}{E-E_{\gamma}\left(k^{\prime \prime}\right)+i \epsilon} \\
& \quad \times t_{\gamma \beta}\left(\mathbf{k}^{\prime \prime}, \mathbf{k}^{\prime} ; E\right) d \mathbf{k}^{\prime \prime}
\end{aligned}
$$

where

$$
E_{\gamma}\left(k^{\prime \prime}\right)=\left(M_{\gamma}{ }^{2}+k^{\prime \prime 2}\right)^{1 / 2}+\left(m_{\gamma}{ }^{2}+k^{\prime \prime 2}\right)^{1 / 2}
$$

is the energy of the virtual meson-baryon system in the intermediate state. The expansion in partial waves is made in the usual way:

$$
t_{\alpha \beta}\left(\mathbf{k}, \mathbf{k}^{\prime} ; E\right)=\sum_{l, m} Y_{l m}(\hat{k}) Y_{l m}^{*}\left(\hat{k}^{\prime}\right) t_{\alpha \beta}^{l}\left(k, k^{\prime} ; E\right)
$$

so that the on-shell, diagonal amplitude is related to the phase shift $\left(\delta_{l}^{\alpha}\right)$ by $^{30}$

$$
t_{\alpha \alpha}^{l}(E)=t_{\alpha \alpha}^{l}\left(k_{\alpha}, k_{\alpha} ; E\right)=-\frac{e^{i \delta_{l}^{\alpha}} \sin \delta_{l}^{\alpha}}{\pi \mu_{\alpha} k_{\alpha}} .
$$

In order to avoid the singularity in the denominator of Eq. (2.45) for $E=E_{\gamma}\left(k^{\prime \prime}\right)$, we do a principal-value subtraction. ${ }^{31}$ That is, we use the fact that

$$
\mathrm{P} \int_{0}^{\infty} \frac{d k^{\prime}}{k^{2}-k^{\prime 2}}=0 \text {, }
$$

to subtract the quantity

$$
2 k^{2} \mu(k) v(k, k ; E) t(k, k ; E) /\left(k^{2}-k^{\prime 2}\right) \text {. }
$$

This produces a smooth integrand for which it is no longer necessary to calculate a principal-value integral. Then we solve the equation in matrix form by doing the integrations by Gaussian quadrature.

\section{1. $K N S$-wave scattering}

For $K N$ scattering the potential Eq. (2.44) reduces to the contact term, because for $S$ wave there is no candidate with strangeness +1 for the state $\left|B_{0}^{\prime}\right\rangle$. Hence the potential for isospin-I, $S$-wave scattering reduces to Eqs. (2.25) and (2.26) with the coupling constants given in Table I. This table contains the unrenormalized coupling constants extracted under our working hypothesis of exact $\mathrm{SU}(3)_{F}$ symmetry. If we take into account the fact that the renormalizations for $K^{+} p$ and $K^{+} n$ are different (using the renormalization procedure explained in Sec. II A) there is a small $I=0$ coupling. For example, for a bag radius of $1 \mathrm{fm}$ the renormalization factors are $\mathscr{R}^{K+}=0.99$ and $\mathscr{R}^{K+}=1.03$ (see Table III), which leads to an $I=0$ coupling around $5 \%$ of that in $I=1$.

By iterating this potential in a Lippmann-Schwinger equation we get the scattering amplitude corresponding to the series shown in Fig. 3. For $K N$ scattering there are only two free parameters, namely, the meson decay constant $f$ and the bag radius. The radius dependence of the $I=1$ scattering length is shown in Fig. 4 for two values of $f$. Our results are consistent with the data indicated by the dashed region in this figure. It is worth remembering that the renormalization of the coupling constant for $K^{+} p$ increases the effective value of the meson decay constant by $1 \%$. Hence, assuming a starting value of 93 $\mathrm{MeV}$, the effective value is $94 \mathrm{MeV}$. Assuming an average value between $f_{\pi}$ and $f_{K}$, namely, $103 \mathrm{MeV}$, the renormalized value is $104 \mathrm{MeV}$. Either value would give a good description of the data. The $I=0$ scattering length is $a_{0} \simeq a_{1} / 20 \simeq-0.01 \mathrm{fm}$, while the experimental values 


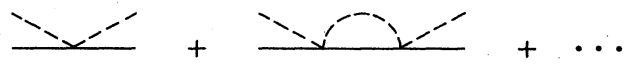

FIG. 3. Series generated by the contact term. Baryons are represented by solid lines and mesons by dashed ones.

vary between $-0.11 \mathrm{fm}$ and $0.04 \mathrm{fm},{ }^{32} a_{0}=+0.02 \mathrm{fm}$ being the most recent one. ${ }^{33}$ Although our result is consistent with existing data, we do not claim to give a good description of the $I=0$ scattering length because, as it is very small, a slight admixture of $I=1$ coming through crossed graphs can be relevant. This comparison is interesting mainly to give an idea of the small effect of higher-order terms not taken into account in the present model.

The mass of the strange quark does not affect the results presented here because this quark is not directly involved in the $K N \rightarrow K N$ transition.

\section{S-wave pion-nucleon scattering}

As an additional check on the model we have also calculated the $S$-wave $\pi N$ scattering length. Just the contact term has been included because the lowest two possible states which contribute to the separable term, namely, the $N(1520)$ and the $\Delta(1620)$, are already more than $400 \mathrm{MeV}$ above threshold. The isospin $\frac{1}{2}$ and $\frac{3}{2}$ scattering lengths produced by the Born contact term are $a_{1}=0.22 \mathrm{fm}$ and $a_{3}=-0.11 \mathrm{fm}$, respectively, for $R=1 \mathrm{fm}$ and $f=93$ $\mathrm{MeV}$. This is in reasonable agreement with the experimental results, ${ }^{34} a_{1}=0.240 \mathrm{fm}$ and $a_{3}=0.145 \mathrm{fm}$. Unfortunately this agreement seems somewhat fortuitous. From the renormalization argument we expect $f$ to be effectively increased by $3 \%$, which changes these values to $a_{1}=0.20 \mathrm{fm}$ and $a_{3}=-0.10 \mathrm{fm}$. Another point is that the rescattering increases the value of $a_{1}$ and decreases $a_{3}$ (it becomes less negative), so that the isospin averaged value $a_{1}+2 a_{3}$ becomes nonzero. For example, using $f=100 \mathrm{MeV}$ and $R=1 \mathrm{fm}, a_{1}=-0.33 \mathrm{fm}$ and $a_{3}=-0.08 \mathrm{fm}$.

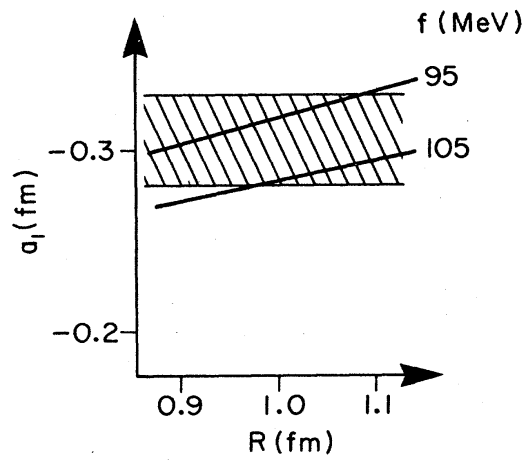

FIG. 4. The $I=1 K N$ scattering length plotted against the bag radius for different values of $f$. The shaded region indicates the range of experimental results, $a_{1}=-0.33 \mathrm{fm}$ being the more recent one (Ref. 33).
The reason for the disagreement is not obvious. One possibility is the $Z$ or antiparticle graphs. However, in agreement with Brodsky ${ }^{35}$ we would argue that such graphs are suppressed by the finite size of the nucleon. Another possibility is that the multiple scattering is suppressed by the finite size of the pion, as suggested by Crawford and Miller. ${ }^{36}$ However, this effect is much too small. A more likely possibility is that the pion interacts with the bag itself. Certainly excluding the pion from the bag would generate much too large a repulsion. Introducing a weak repulsion for the pion inside the bag would, however, cure the problem. Since this leads too far from our main concern of $\bar{K} N$ scattering it will not be considered further here.

\section{THE COUPLED $\bar{K} N-\Sigma \pi$ SYSTEM IN THE VICINITY OF THE $\Lambda^{*}(1405)$}

To describe $\bar{K} N$ scattering we include the $\bar{K} N, \pi \Sigma$, and $\pi \Lambda$ channels as well as a $\Lambda^{*}$ bare three-quark state. For the case of scattering there are several three-quark states which can in principle contribute to the separable part of the potential. However, for the volume coupling version of the CBM the higher excited states give a relatively small contribution for low-energy scattering. ${ }^{37}$ Therefore, we restrict the calculation to just one excited baryon state. As a rough guide to the structure of the lowest $\frac{1}{2}^{-} \Lambda^{*}$ state, we note that Isgur and $\mathrm{Karl},{ }^{38}$ in their nonrelativistic quark model, find that the lowest $\frac{1}{2}^{-} \Lambda^{*}$ state is $80 \%$ an SU(3) singlet with the rest being octet. In our calculation we take the bare state to be a pure $S U(3)$ singlet. Since we find that the $\Lambda^{*}(1405)$ is predominantly a $\bar{K} N$ bound state, we do not expect the details of the bare state to be important.

The potential for the $\bar{K} N S$-wave scattering [Eq. (2.44)] reduces to just one separable potential given by Eqs. (2.2) and (2.22), and a contact part given by Eqs. (2.25) and (2.26). The coupling constants are given in Table III. This potential is iterated in the relativistic LippmannSchwinger Eq. (2.45) producing the scattering amplitude corresponding to the graphs shown in Figs. (3) and (5).

\section{A. Scattering in $S$ wave}

In the case of $S$-wave $\bar{K} N$ scattering our model has three parameters, namely, $f, R$, and $M_{0}$ (the mass of the bare $\Lambda^{*}$ state). These parameters are adjusted in order to

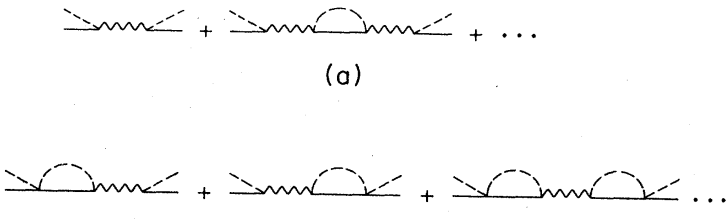

(b)

FIG. 5. (a) Series generated by the separable potential and (b) its interference with the contact term. Baryon-octet members are represented by solid lines, mesons by dashed ones, and the $\Lambda^{*}$ by wiggly lines. 
get a reasonable $\pi \Sigma$ mass spectrum compared to the data. For most of the discussion we will take the strange quark to be massless since we have checked that the mass has much less effect than other uncertainties in the calculation. The thresholds are taken to be 1432.6, 1331.6, and $1254.6 \mathrm{MeV}$ for $\bar{K} N, \pi \Sigma$, and $\pi \Lambda$, respectively. When the nondegeneracy of the $K^{-} p$ and $\bar{K}^{0} n$ is included, the $\bar{K}^{0} n$ threshold is $1437.3 \mathrm{MeV}$.

In Fig. 6 we compare our results with the $\pi \Sigma$ mass spectrum measured by Hemingway et al. ${ }^{27}$ for $K^{-} p \rightarrow \pi^{+} \pi^{-}\left(\Sigma^{-} \pi^{+}\right)$. The theoretical curves are $k_{\mathrm{c} . \mathrm{m} .}^{\pi}\left|T_{\pi \Sigma}\right|^{2}$. The normalization is arbitrary, since we are interested just in the shape of the spectrum. The solid and dashed curves correspond to $(R, f)$ equal to $(1.0 \mathrm{fm}$, $120 \mathrm{MeV})$ and $(1.1 \mathrm{fm}, 110 \mathrm{MeV})$, respectively. The values for $f$ are larger than those obtained with the renormalization procedure. However the mass spectrum is very sensitive to this parameter and it is not possible to get a reasonable mass spectrum without increasing the value of $f$. The bare mass of the $\Lambda^{*}$ turns out to be $M_{0}=1630$ $\mathrm{MeV}$ for the set $(1.0 \mathrm{fm}, 120 \mathrm{MeV})$ and $M_{0}=1650 \mathrm{MeV}$ for the other set. Variations in these masses around \pm 5 $\mathrm{MeV}$ are acceptable (we note that in Ref. 26) the bare masses were different because our results were compared with older data). In this comparison we took just the $I=0$ piece. [The $I=1 \Sigma \pi$ interaction is less attractive in the $S$ wave (Table I), and the effect of the $\Sigma^{*}(1385)$ is suppressed by an angular momentum barrier.] In Fig. 7 we show the $I=0$ scattering amplitude for the two sets of parameters. The real piece stays high very near the $\pi \Sigma$ threshold, showing that the cutoff in the mass spectrum at low energy comes from the phase-space factor $k_{\text {c.m. }}^{\pi}$ by which $\left|T_{\pi \Sigma}\right|^{2}$ is multiplied.

The $\bar{K} N$ elastic-scattering amplitude can be seen in Fig. 8 for $I=0$. We note a rapid variation with the energy near the $\bar{K} N$ threshold, which may be useful in reconciling the kaonic-hydrogen energy shift with the scattering data.

The effective value for $f$ in the $I=1$ piece is kept inside limits compatible with the renormalization procedure, using $f_{I=1}^{\bar{K} N}$ smaller than $f_{I=1}^{\pi \Sigma}$. This gives for

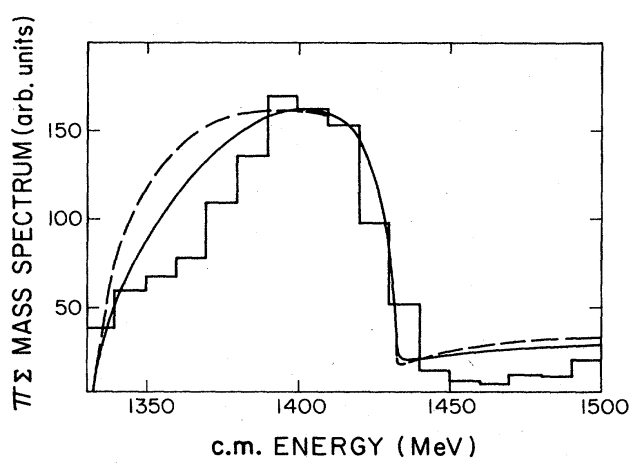

FIG. 6. The $\pi \Sigma$ mass distribution. The histogram is data from Hemingway et al. (Ref. 27). The theoretical curves are $k_{\mathrm{c} . \mathrm{m} .}^{\pi}\left|T_{\pi \Sigma}\right|^{2}$. The solid curve corresponds to parameter set A and the dashed curve to parameter set $B$.
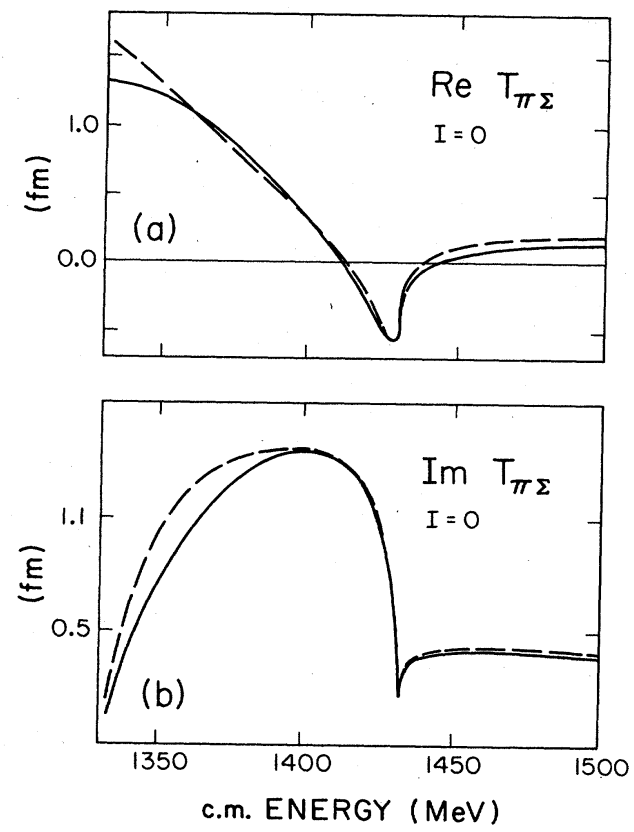

FIG. 7. The $\pi \Sigma$ elastic-scattering amplitude corresponding to parameter set A (solid curve) and B (dashed curve).

$\left(R, f_{\pi \Sigma, \pi \Lambda}, f_{\bar{K} N}\right)$ the values $(1.0,110,100)$ and $(1.1,105$, 95). The $\bar{K} N$ scattering amplitudes for these two sets of parameters are shown in Fig. 8.

To summarize, our two sets of parameters are as follows.

Set A:

$$
\begin{aligned}
& R=1.0 \mathrm{fm}, M_{0}=1630 \mathrm{MeV} \\
& f^{I=0}=120 \mathrm{MeV}, f_{\pi \Sigma, \pi \Lambda}^{I=1}=110 \mathrm{MeV} \\
& f_{\bar{K} N}^{I=1}=100 \mathrm{MeV}
\end{aligned}
$$
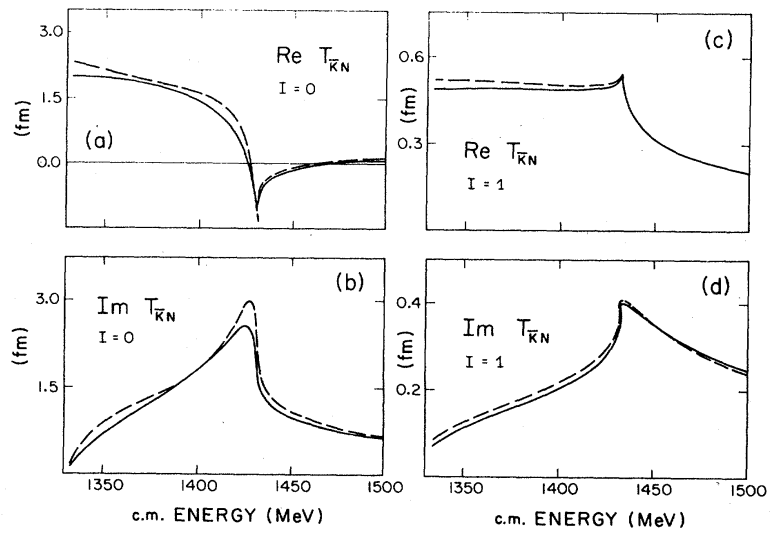

FIG. 8. The $\bar{K} N$ elastic-scattering amplitude for $I=0$ (a) and (b) and $I=1,(\mathrm{c})$ and (d), with the parameter set A (solid curve) and $B$ (dashed curve). 
Set B:

$$
\begin{aligned}
& R=1.1 \mathrm{fm}, M_{0}=1650 \mathrm{MeV}, \\
& f^{I=0}=110 \mathrm{MeV}, f_{\pi \bar{\Sigma}, \pi \Lambda}^{I=1}=105 \mathrm{MeV}, \\
& f_{\bar{R} N}^{I=1}=95 \mathrm{MeV} .
\end{aligned}
$$

The comparison between the theoretical and experimental cross sections $s^{39,40}$ is made in Fig. 9. In these cross sections we include the Coulomb correction and the effect of the nondegenerate masses of the $K^{-} p$ and $\bar{K}^{0} n$ as derived by Dalitz and Tuan. ${ }^{41}$ We note that Evans et al. ${ }^{42}$ claim a contribution from $p$ waves for the charge-exchange process at an incident momentum around $230 \mathrm{MeV} / c$, and for $\sigma$ production at a momentum as low as $150 \mathrm{MeV} / c$.

\section{B. The scattering lengths}

Table IV contains the $\bar{K} N$ scattering lengths for the two sets of parameters, taking massless $u$ and $d$ quarks, and including the mass of the strange quark (300 MeV). (The mass of the strange quark is introduced in the manner shown in Appendix A.) The $u$ and $d$ quarks are kept massless because even the large mass of the $s$ quark has a small effect. (Its effect on the cross sections presented is smaller than $3 \%$.) In the $\pi \Sigma$ mass spectrum, the $s$-quark mass tends to decrease the bare mass of the $\Lambda^{*}$ by 5 or 6 $\mathrm{MeV}$.
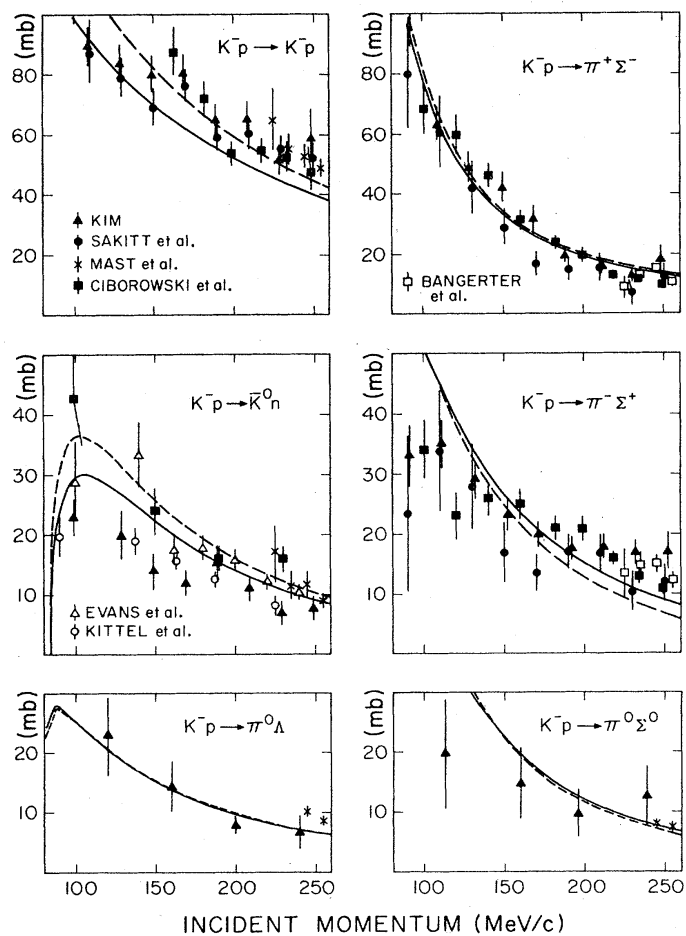

FIG. 9. Comparison of the $K^{-} p$ cross sections at low energy with data from Refs. 39 and 40 . The solid line corresponds to parameter set $\mathbf{A}$ and the dashed one to parameter set $\mathbf{B}$.
TABLE IV. The $\bar{K} N$ scattering length for the two sets of parameters taking all the quarks massless and the $s$ quark with mass $\left(\boldsymbol{M}_{\boldsymbol{s}}\right)$.

\begin{tabular}{cccc}
\hline \hline Parameter set & $M_{s}(\mathrm{MeV})$ & $a_{0}(\mathrm{fm})$ & $a_{1}(\mathrm{fm})$ \\
\hline$A$ & 0 & $-1.14+1.76 i$ & $0.53+0.39 i$ \\
$B$ & 0 & $-1.36+2.07 i$ & $0.54+0.40 i$ \\
$A$ & 300 & $-1.02+1.97 i$ & $0.55+0.42 i$ \\
$B$ & 300 & $-1.22+2.30 i$ & $0.56+0.44 i$ \\
\hline \hline
\end{tabular}

To compare these scattering lengths with those extracted from the kaonic-hydrogen energy shift $\left[a_{\bar{K} p}=(0.10\right.$ $\pm 0.15)+(0 \pm 0.28) i \mathrm{fm}]^{42}$ it is necessary to include the splitting of the $\bar{K} p$ and $\bar{K}^{0} n$ masses and a Coulomb correction. The mass splitting changes the real piece of the average scattering length $\bar{a}=\frac{1}{2}\left(a_{0}+a_{1}\right)$, e.g., for set $\mathrm{A}$, from -0.31 to $-0.26 \mathrm{fm}$. Including the Coulomb correction just outside the range of the nuclear interaction, ${ }^{41}$ this value is shifted to $-0.38 \mathrm{fm}$. It is possible to improve the Coulomb correction, ${ }^{43}$ however it is not likely that it can have so large an effect as to reconcile our results with the kaonic-hydrogen data.

For $\bar{K} N$ elastic scattering the Born term involving crossed meson lines vanishes by strangeness conservation. Hence its effect comes only in higher order, and as a consequence has a small effect on the scattering length. For example, including this kind of graph the scattering length for parameter set $\mathrm{A}$ is $a_{0}=-1.02+1.74 i \mathrm{fm}$ and $a_{1}=-0.54+0.38 i \mathrm{fm}$.

\section{DISCUSSION OF THE RESULTS}

The $S$-wave $K N$ scattering is the cleanest process studied in this paper. As soon as there is no baryon with $S=+1$ to be included there is only the "freedom" to vary $f$ and $R$. Happily it seems that the values of $f$ and $R$ which describe the data are quite consistent with the renormalization and radius expected in the CBM.

The agreement between experiment and theory for $\pi N$ scattering is not as good. The contact term gives a contribution quantitatively similar to the $\rho$ exchange included in more conventional models. ${ }^{44,45}$ (As pointed out many years ago by Weinberg ${ }^{46}$ this is not entirely accidental.) To get better agreement with the data we clearly need some more physics. One might consider some of the standard phenomenological suggestions ${ }^{45}$ such as a hard core, or $Z$ graphs. These contributions are outside the scope of the present paper, and we only note that at this stage, the CBM does not seem to cast a new light on the problem.

The $S$-wave $\bar{K} N$ system has the additional complication of an intermediate state, the $\Lambda^{*}(1405)$. This state raises problems in both the nonrelativistic quark model ${ }^{38}$ and in the MIT bag model. ${ }^{47}$ In the nonrelativistic quark model it comes too high in energy and is degenerate with the $\frac{3}{2}^{-}$ $\Lambda^{*}$ state which experimentally is at $1520 \mathrm{MeV}$. In the bag model the $\frac{1}{2}^{-}$state occurs higher than the $\frac{3}{2}^{-}$due to the spin-orbit force.

Our model of the $\Lambda^{*}(1405)$ is very similar to that of Dalitz, Wong, and Rajasekaran. ${ }^{25}$ In that calculation the 
potential was taken from vector-meson exchange with the relative coupling constants taken from SU(3) symmetry. The range of potential was determined by the masses of the vector mesons. In the present calculation the contact interaction has the same spin-flavor structure as the vector mesons, namely, the quark part of the coupling is

$$
\bar{q} \gamma^{\mu} \lambda^{a} q \theta_{v}
$$

the meson part is

$$
f_{a b c} \phi_{b} \partial_{\mu} \phi_{c}
$$

and the coupling constants given in Table I for the contact interaction are in fact the same as those given by Dalitz and co-workers ${ }^{25}$ (apart from the phase convention). In the present case the range of the potential is given by the bag radius $R$ rather than the vector-meson masses. The net result will be somewhat similar. The main difference is that in our model elementary states (i.e., threequark states) appear in a rather natural way and it is the model that decides to what extent the $\Lambda^{*}(1405)$ is a $\bar{K} N$ bound state or a normal quark state. We find that the $\Lambda^{*}(1405)$ is predominantly a $\bar{K} N$ bound state with only $14 \%$ of the strength in the $\pi \Sigma$ cross section around 1405 $\mathrm{MeV}$ coming from the bare quark state-see Appendix B. We also have a higher state which presumably corresponds to the $\Lambda^{*}(1670)$. In agreement with previous analysis $^{33}$ we find that the $\Lambda^{*}(1405)$ is not well described by a Breit-Wigner resonance shape.

The $D$-wave scattering will be less strongly affected by the potential because of the centrifugal barrier and we do not expect a $\bar{K} N$ bound state in that channel, although the $\frac{3}{2}$ state may be shifted down somewhat from its unperturbed value. Thus we would claim that the lowest excited three-quark state seen is the $\frac{3}{2}^{-} \Lambda^{*}(1520)$, in agreement with the order suggested by the MIT bag model.

Kumar and Nogami ${ }^{48}$ have proposed that a CastillejoDalitz-Dyson (CDD) zero in the scattering amplitude would reconcile the scattering and kaonic-hydrogen data. Their model has a pole term and a separable contact potential. The main weakness of this model so far, is that it has not been adjusted to reproduce any experimental data. In our model we also have a CDD zero, however it comes out at much too high an energy to explain the discrepancies at threshold. In Fig. 10 we show the inverse $K$ matrix elements and the determinant of the inverse $K$ matrix. The pole in the determinant at about $1700 \mathrm{MeV}$ is the CDD pole. The inverse $K$ matrix elements, shown over a more restricted energy interval, indicate that these elements do indeed have a linear dependence over a considerable energy range.

The $\bar{K} N$ scattering amplitude given by the CBM shows a strong energy dependence near threshold. It is worth noting that as a consequence of the coupled channel nature of the problem the resonance structure in the $\pi \Sigma$ elastic-scattering amplitude appears in a different position than the change in sign in the $\bar{K} N$ scattering amplitude. In particular, the former occurs at ground $1410 \mathrm{MeV}$ (Fig. 7), while the latter occurs near $1428 \mathrm{MeV}$ (Fig. 8). To explain the disagreement between scattering and kaonichydrogen data it would be necessary to move the zero in the $\bar{K} N$ case something like $5 \mathrm{MeV}$ above threshold
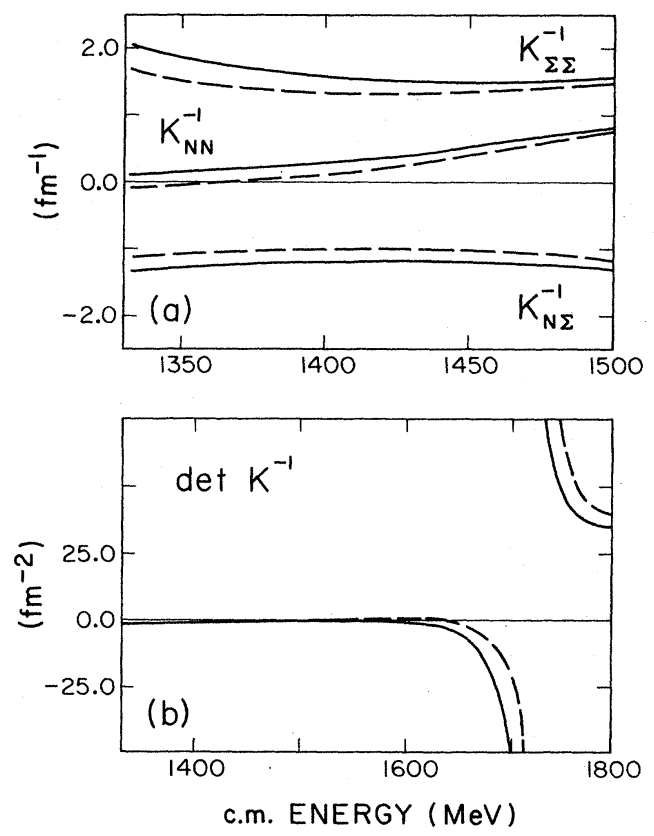

FIG. 10. (a) Matrix elements of the inverse $K$ matrix and (b) the determinant of the inverse $K$ matrix $(I=0)$.

without spoiling the $\pi \Sigma$ mass spectrum. However, we were not able to do this with reasonable sets of parameters.

Our results for the $\bar{K} N$ scattering length differ in magnitude from values quoted in the literature (e.g., $a_{0}=1.70+0.68 i \mathrm{fm}$ and $\left.a_{1}=0.37+0.60 i \mathrm{fm}\right),{ }^{25}$ but with respect to the sign favor the scattering data.

\section{CONCLUSIONS}

The CBM has been extended to chiral SU(3) $\times \mathrm{SU}(3)$ and applied to the problem of kaon-nucleon scattering. First some comments must be made on the limitations of the model. We use a static, spherical bag with a sharp surface, so that the deformation of the $\Lambda^{*}(1405)$ is neglected. We also ignore any center-of-mass correction and use the same radius for all baryons. Some of these limitations can in principle be improved ${ }^{49}$ but only at the cost of greatly complicating the calculation. Beyond that, it is not clear how to include the center-of-mass correction. Although interesting, these effects can hardly change the conclusions of the present work.

Crawford and Miller ${ }^{36}$ have shown that by using the finite size of the pion, the contribution of excited quark states to the self-energy is finite and small. This explains why the self-energies do not diverge and leads to the conclusion that it is safer when using pointlike mesons to not include a complete set of excited quark states which may yield unphysical results. Additionally, the extended meson decreases the multiple scattering.

In the present model we use pointlike mesons, but do not allow quark excitations beyond the $1 p_{1 / 2}$ level. Thus we do not include those contributions which would anyway be cutoff by smearing the field in space and time. As 
a first step we have considered only $s$-wave scattering, which restricts our comparison with the data to very low energy. Clearly the next step is to extend the calculation to other partial waves. The comparison of our results with data for low-energy $K N$ and $\bar{K} N$ scattering is quite good. Thus, in spite of the large mass of the strange quark, and the badly broken SU(3) symmetry in nature, the SU(3) CBM seems to make sense as soon as the physical masses are taken into account. The $\bar{K} N$ scattering length which we find disagrees with that extracted from $\bar{K} p$ atom data, as do all the scattering lengths extracted from scattering data. The remaining point to be checked is whether by using the strong-interaction potential produced by the CBM directly in the kaonic-hydrogen calculations it is possible to get the energy shift measured in the kaonic-hydrogen experiments. Work is in progress on this problem. 50

The results obtained for low-energy $\pi N$ scattering are not as good. However, the contact term gives a contribution similar to the $\rho$ meson in meson-exchange descriptions of $\pi N$ scattering. It is worth commenting at this point that the contact term has many features in common with the exchange of an octet of vector mesons. As pointed out many years ago by Weinberg, ${ }^{46}$ the vector mesons can be introduced explicitly in the covariant derivatives that appear in the volume coupling. While that is probably inconsistent with the present model, it does indicate that many effects which are traditionally ascribed to vector mesons may be equally well described by the contact term.

In the controversy over the nature of the $\Lambda^{*}(1405)$, the present model comes down firmly on the side of the $\Lambda^{*}(1405)$ being primarily a $\bar{K} N$ bound state. ${ }^{25}$ This indicates that the $\Lambda^{*}(1405)$ should not be included as one of the states fit in simple quark-model descriptions of baryon resonances.

In summary, the CBM provides a useful description of kaon-nucleon scattering at low energy in spite of the fact that $\mathrm{SU}(3)$ symmetry is so badly broken in nature.

\section{ACKNOWLEDGMENTS}

It is a pleasure to acknowledge stimulating and useful conversation with E. Ferreira, R. Hemingway, G. Karl, O. V. Maxwell, D. J. Miller, G. A. Miller, J. M. Richard, A. Rinat, R. H. Landau, and N. de Takacsy. Natural Sciences and Engineering Research Council (Canada), Conselho Nacional de Pesquisas do Brasil and Financiadora de Estudos e Projectos (Brazil), Science and Engineering Research Council (United Kingdom), CERN, and The University of Adelaide are thanked for financial support.

\section{APPENDIX A}

In this appendix we present the modifications to the formalism required by the massive strange quark. The $u$ and $d$ quarks are kept massless. It would not be hard to include their masses but since even the large mass of the strange quark has little effect there is no reason to. The $1 s_{1 / 2}$ and $1 p_{1 / 2}$ massive-quark wave functions are given, respectively, by [instead of Eq. (2.16) and (2.17)] $q_{1 s}(\mathbf{r}, t)=\frac{N_{s}^{\prime}}{\sqrt{4 \pi}}\left[\begin{array}{c}\alpha_{s}^{+} j_{0}\left(w_{s}^{\prime} r\right) \\ i \alpha_{s}^{-} \boldsymbol{\sigma} \cdot \hat{\mathbf{r}} j_{1}\left(w_{s}^{\prime} r\right)\end{array}\right] e^{-i w_{s}^{\prime} t} b \theta(R-r)$

and

$q_{1 p_{1 / 2}}(\mathbf{r}, t)=\frac{N_{p}^{\prime}}{\sqrt{4 \pi}}\left(\begin{array}{c}\alpha_{p}^{+} \boldsymbol{\sigma} \cdot \hat{\mathbf{r}} j_{1}\left(w_{p}^{\prime} r\right) \\ i \alpha_{p}^{-} j_{0}\left(w_{p}^{\prime} r\right)\end{array}\right) e^{-i w_{p}^{\prime} t} b \theta(R-r)$,

where

$$
\alpha_{s, p}^{ \pm}=\left(\frac{\alpha_{s, p} \pm m R}{\alpha_{s, p}}\right)^{1 / 2} .
$$

The corresponding normalization constants are given by

$$
N_{s, p}^{\prime}=\frac{1}{j_{0}{ }^{2}\left(w_{s, p}^{\prime} R\right) R^{3}} \frac{\alpha_{s, p}\left(\alpha_{s, p} \mp m R\right)}{2 \alpha_{s, p}\left(\alpha_{s, p}-1\right)+m R},
$$

where

$$
\alpha_{s, p}=\left[\left(w_{s, p}^{\prime} R\right)^{2}+(m R)^{2}\right]^{1 / 2} .
$$

The energies are $w_{s}^{\prime}=2.51 \cdots / R$ and $w_{p}^{\prime}=3.96 \cdots / R$ for $m R=300 \mathrm{MeV}$.

The strange-quark mass affects the matrix elements of $H_{s}$ and $H_{c}$ in different ways. We consider the case corresponding to $H_{s}$ first. The massive quark invalidates Eq. (2.12) because that equation was derived using the Dirac equation for massless quarks. When the mass of the strange quark is taken into account the first-order interaction Hamiltonian changes to

$$
\hat{H}_{s}^{M}=\hat{H}_{s}+\frac{i}{f} \int d^{3} x \theta_{v} \bar{q} \gamma_{5} \lambda q \cdot \phi .
$$

Furthermore, the mass affects the normalization and energy of the quark state as well as the relative weight between upper and lower components of the quark wave function. These effects only modify transitions which involve a strange quark directly, namely, the $\bar{K} N \rightarrow \Lambda^{*}$ transition. (There is no effect on the $\pi \Sigma \rightarrow \Lambda^{*}$ transition.) The form factor for $\bar{K} N \rightarrow \Lambda^{*}$ is given by [instead of Eq. (2.22)]:

$$
\begin{aligned}
& u_{N \bar{K} \Lambda^{*}}(k, R)=N_{s} N_{s}^{\prime}\{ 2 R^{2} \alpha_{p}^{-} j_{0}\left(w_{s} R\right) j_{0}\left(w_{p}^{\prime} R\right) j_{0}(k R) \\
&-\left[w_{s}-w_{p}^{\prime}+w_{\bar{K}}(k)\right] \int_{0}^{R} d x F_{+}(k x) \\
&\left.+m \int_{0}^{R} d x F_{-}(k x)\right\},
\end{aligned}
$$

where

$$
F_{ \pm}(k, x)=x^{2}\left[\alpha_{p}^{-} j_{0}\left(w_{s} x\right) j_{0}\left(w_{p}^{\prime} x\right)\right.
$$

$$
\left.\pm \alpha_{p}^{+} j_{1}\left(w_{s} x\right) j_{1}\left(w_{p}^{\prime} x\right)\right] j_{0}(k x) \text {. }
$$

The quantities with primes refer to the massive strange quark and those without primes to the massless quarks.

With respect to $H_{c}$ the mass of the strange quark affects the $\bar{K} N \rightarrow \pi \Sigma$ and the $\bar{K} N \rightarrow \pi \Lambda$ transitions, without any effect on the elastic transitions. Thus the form factor changes to 


$$
\begin{aligned}
u_{\alpha \beta}^{\prime}\left(k, k^{\prime}, R\right)= & N_{s} N_{s}^{\prime}\left[w_{\alpha}(k)+w_{\beta}\left(k^{\prime}\right)\right] \\
& \times \int_{0}^{R} d x x^{2}\left[\alpha_{s}^{+} j_{0}\left(w_{s} x\right) j_{0}\left(w_{s}^{\prime} x\right)\right. \\
& \left.+\alpha_{s}^{-} j_{1}\left(w_{s} x\right) j_{1}\left(w_{s}^{\prime} x\right)\right] \\
& \times j_{0}(k x) j_{0}\left(k^{\prime} x\right),
\end{aligned}
$$

where $\alpha$ stands for $\bar{K} N$ and $\beta$ for $\pi \Sigma$ or $\pi \Lambda$.

\section{APPENDIX B}

We would like to estimate the relative importance of the bare $\Lambda^{*}$ and the contact interaction at the pole of the $\pi \Sigma t$ matrix. Rather than actually doing the calculation at the complex energy where the pole occurs we actually stay on the real axis at $1410 \mathrm{MeV}$. Using the fact that the pole is associated with the last term in the LippmanSchwinger equation,

$$
t=v+v G_{0} t
$$

we estimate the relative contribution by comparing $v_{\text {pole }} G_{0} t$ with $v_{\text {con }} G_{0} t$, where $v_{\text {pole }}$ and $v_{\text {con }}$ are, respective$1 \mathrm{y}$, the first and second terms on the right-hand side of Eq. (2.44). As noted in the text the pole term gives about $14 \%$ of the total width.
*Permanent address: Universidade Federal do Rio Grande do Sul, Rua Luiz Englert, s/n, 90000, Porto Alegre, Brazil.

${ }^{1}$ T. Inoue and T. Maskawa, Prog. Theor. Phys. 54, 1833 (1975); A. Chodos and C. B. Thorn, Phys. Rev. D 12, 2733 (1975).

${ }^{2}$ G. E. Brown and M. Rho, Phys. Lett. 82B, 177 (1979).

${ }^{3}$ R. L. Jaffe, in Pointlike Structures Inside and Outside Hadrons, proceedings of the Seventeenth International School of Subnuclear Physics, Erice, 1979, edited by A. Zichichi (Plenum, New York, 1982), p. 99.

${ }^{4}$ A. W. Thomas, in Advances in Nuclear Physics, edited by $\mathrm{J}$. Negele and E. Vogt (Plenum, New York, 1984), Vol. 13, p. 1.

${ }^{5} \mathrm{G}$. E. Brown, in Progress in Particle and Nuclear Physics, edited by D. Wilkinson (Pergamon, London, 1982), Vol. 8, p. 147.

${ }^{6}$ C. E. De Tar and J. F. Donoghue, Annu. Rev. Nucl. Part. Sci. 33, 235 (1983).

${ }^{7} \mathrm{~A}$. W. Thomas, in Mesons, Isobars, Quarks and Nuclear Excitations, proceedings of the International School of Subnuclear Physics, Erice, 1983 [Progress in Particle and Nuclear Physics, edited by D. Wilkinson (Pergamon, London, 1984), Vol. 11].

${ }^{8}$ T. H. R. Skyrme, Proc. R. Soc. London A260, 127 (1961).

${ }^{9}$ M. Rho, A. S. Goldhaber, and G. E. Brown, Phys. Rev. Lett. 51, 747 (1983); J. Goldstone and R. Jaffe, ibid. 51, 1518 (1983).

${ }^{10}$ E. Witten, Nucl. Phys. B160, 57 (1979); B223, 422 (1983); B223, 433 (1983).

${ }^{11}$ S. T. Théberge and A. W. Thomas, Nucl. Phys. A393, 252 (1983).

${ }^{12}$ F. Myhrer, G. E. Brown, and Z. Xu, Nucl. Phys. A362, 317 (1981).

${ }^{13}$ C. De Tar, Phys. Rev. D 24, 752 (1981); 24, 764 (1981).

${ }^{14}$ A. W. Thomas, S. Théberge, and G. A. Miller, Phys. Rev. D 24, 216 (1981); 22, 2838 (1980); 23, 2106(E) (1981).

${ }^{15}$ W. Weise, in Mesons, Isobars, Quarks, and Nuclear Excitations (Ref. 7).

${ }^{16}$ R. Tegen, M. Schedl, and W. Weise, Phys. Lett. 125B, 9 (1983).

17P. A. M. Guichon, G. A. Miller, and A. W. Thomas, Phys. Lett. 124B, 109 (1983).

${ }^{18}$ B. Loiseau, N. Cottingham, and O. Maxwell, report, 1983 (unpublished).

${ }^{19}$ A. Szymacha and S. Tatur, Z. Phys. C 7, 311 (1981).

${ }^{20}$ A. W. Thomas, J. Phys. G 7, L283 (1981).

${ }^{21}$ G. Kalbermann and J. M. Eisenberg, Phys. Rev. D 28, 66 (1983).

22P. Zenczykowski, Phys. Rev. D 29, 577 (1984).

23P. Gonzalez and V. Vento, Nucl. Phys. A407, 349 (1983).

${ }^{24}$ C. J. Batty, Nukleonica 25, 545 (1980).
${ }^{25}$ R. H. Dalitz and J. G. McGinley, in Low and Intermediate Energy Kaon-Nucleon Physics, edited by E. Ferrari and G. Violini (Reidel, Boston, 1981), p. 381; R. H. Dalitz, T. C. Wong, and G. Rajasekaran, Phys. Rev. 153, 1617 (1967).

${ }^{26}$ E. A. Veit, B. K. Jennings, R. Barrett, and A. W. Thomas, Phys. Lett. 137B, 415 (1984).

${ }^{27}$ R. Hemingway (private communication).

${ }^{28}$ See, for example, J. J. Sakurai, Currents and Mesons (University of Chicago Press, Chicago, 1969).

${ }^{29}$ A. J. G. Hey, in Topics in Quantum Field Theory and Gauge Theories, proceedings of the Eighth International Seminar on Theoretical Physics, Salamanca, Spain, 1977, edited by J. A. de Azcarraga (Lecture Notes in Physics 77) (Springer, Berlin, 1978), p. 155.

${ }^{30}$ M. L. Goldberger and K. M. Watson, Collision Theory (Wiley, New York, 1964).

${ }^{31}$ M. I. Haftel and F. Tabakin, Nucl. Phys. 158, 1 (1970).

${ }^{32}$ C. B. Dover and G. E. Walker, Phys. Rep. 89, 1 (1982).

${ }^{33}$ A. D. Martin, Nucl. Phys. B179, 33 (1981).

34J. Stahov, Karlsruhe Report No. TKP82-16 (unpublished).

${ }^{35}$ S. J. Brodsky and J. R. Hiller, Phys. Rev. C 28, 475 (1983); S. J. Brodsky, Short Distance Phenomena in Nuclear Physics, edited by D. H. Boal and R. M. Woloshyn (Plenum, New York, 1983), p. 141.

${ }^{36}$ G. A. Crawford and G. A. Miller, Phys. Lett. 132B, 173 (1983).

${ }^{37}$ B. K. Jennings and O. V. Maxwell, Nucl. Phys. A422, 589 (1984).

${ }^{38}$ N. Isgur and G. Karl, Phys. Rev. D 18, 1 (1982).

${ }^{39}$ M. Sakitt, T. B. Day, R. G. Glasser, N. Seeman, J. Friedman, W. E. Humphrey, and R. R. Ross, Phys. Rev. 139B, 719 (1965); W. Kittel, G. Otter, and I. Wacek, Phys. Lett. 21, 349 (1966); J. K. Kim, Phys. Rev. Lett. 14, 29 (1965); Columbia University Report No. Nevis 149, 1966 (unpublished); Phys. Rev. Lett. 19, 1074 (1967); T. S. Mast, M. Alston-Garnjost, R. O. Bangerter, A. S. Barbaro-Galtieri, F. T. Solmitz, and R. D. Tripp, Phys. Rev. D 11, 3078 (1975); 14, 13 (1976); R. D. Bangerter, M. A. Alston-Garnjost, A. Barbaro-Galtieri, T. S. Mast, F. T. Solmitz, and R. D. Tripp, ibid. 23, 1484 (1981); J. Cibrowski, J. Gwizdz, D. Kielczewska, R. J. Nowak, E. Rondio, J. A. Zakrzewski, M. Godssens, G. Wilquet, N. H. Bedford, D. Evans, G. P. Fleming, Y. A. Hamam, J. V. Major, J. H. Bartley, D. H. Davis, D. J. Miller, D. N. Touee, and T. Tymieniecka, J. Phys. G 8, 13 (1982). The charge-exchange cross section presented in this reference has been corrected in Ref. 40.

${ }^{40}$ D. Evans, J. V. Major, E. Rondio, J. A. Zakrzewski, J. E. 
Conboy, D. J. Miller, and T. Tymieniecka, J. Phys. G 9, 885 (1983).

${ }^{41}$ R. H. Dalitz and S. F. Tuan, Ann. Phys. (N.Y.) 10, 307 (1960).

42J. D. Davies, G. J. Pyle, G. T. A. Squier, C. J. Batty, S. F. Biagi, S. D. Hoath, P. Sharman, and A. S. Clough, Phys. Lett. 83B, 55 (1979).

${ }^{43}$ A. Deloff and J. Law, Phys. Rev. C 21, 2048 (1980).

${ }^{44}$ F. Hachenberg and H. J. Pirner, Ann. Phys. (N.Y.) 112, 401 (1978).
45J. Hamilton, High Energy Physics, edited by E. H. S. Burhop (Academic, New York, 1967), Vol. 1, p. 193; J. Hüfner, Phys. Rep. 21, 1 (1975).

${ }^{46}$ S. Weinberg, Phys. Rev. 166, 1568 (1968).

${ }^{47}$ T. A. DeGrand, Ann. Phys. (N.Y.) 101, 496 (1976).

${ }^{48}$ K. S. Kumar and Y. Nogami, Phys. Rev. D 22, 1834 (1980).

${ }^{49}$ See Ref. 4 and references therein.

${ }^{50}$ R. C. Barrett (private communication); R. H. Landau (private communication). 